\title{
Numerical modeling and analysis of glass fiber drawing process from large sized silica preform
}

\author{
Yong Ki KIM*, Jin Su CHOI**, Ho Sang KWAK*** and Kyoungin KIM*** \\ *Fundamental Technology R\&D Group, Kolon Industries, Inc., \\ 48 Suchul-daero, Gumi, Gyeongbuk 39366, Republic of Korea \\ ${ }^{* *}$ Mobile Communication Business, Samsung Electronics, Gumi, Gyeongbuk, Korea \\ 302 3gongdan 3-ro, Gumi, Gyeongbuk 39388, Republic of Korea \\ ${ }^{* * * D e p a r t m e n t ~ o f ~ M e c h a n i c a l ~ S y s t e m ~ E n g i n e e r i n g, ~ K u m o h ~ N a t i o n a l ~ I n s t i t u t e ~ o f ~ T e c h n o l o g y, ~}$ \\ 61 Daehak-ro, Gumi, Gyeongbuk 39177, Republic of Korea \\ E-mail:kimkj@kumoh.ac.kr
}

Received: 18 July 2017; Revised: 19 September 2017; Accepted: 1 October 2017

\begin{abstract}
As fiberoptics industries have been attempting to use a silica preform of larger diameter in order to reduce facility down time and increase optical fiber manufacturing productivity, this computational study investigates how the use of large sized preform in sized up draw furnace affects high speed glass fiber drawing process. Present computational model for glass fiber drawing simulations employs iterative scheme between one-dimensional momentum balance model for neck-down profile of heated and softened preform and two-dimensional axisymmetric thermo-fluid computations of convective and radiative heat transport for preform heating and purge gas flow inside muffle tube. Prediction of preform neck-down shape for $10 \mathrm{~cm}$ diameter preform has been verified with measured profile from actual optical fiber drawing test. The effects of larger preform diameter up to $20 \mathrm{~cm}$ are appreciated and discussed on temperature distribution of preform and drawn glass fiber and fiber draw tension. This study also shows that fine control of heating condition is necessary to maintain proper amount of draw tension in high precision glass fiber drawing.
\end{abstract}

Keywords: Optical fiber manufacturing, Draw furnace, Glass fiber drawing, Silica preform, Numerical modeling

\section{Introduction}

An optical fiber typically consists of core and cladding layers as a medium for transmitting optical signal along with outer polymer resin coatings as protective layers against microbending and mechanical damages. While the core and cladding layers can be made of several different materials such as sapphire or plastic depending on their specialized applications, silica glass of high purity and high refractive index is the most popular choice of glass fiber material for core and cladding in optical fibers (Richardson et al., 2010). In mass manufacturing of optical fibers, glass fiber drawing from silica preform is an important process and the degree of its process precision could greatly affect the final qualities of produced optical fibers such as superior signal transmission and high fiber strength (Elliott and Gilmore, 2001).

In a draw furnace of optical fiber draw tower, a cylindrical silica preform is slowly fed into the furnace, and then locally heated above glass softening temperature by the heating element of graphite resistance. By applying a proper amount of draw tension on the melted tip of preform, heated and softened preform section experiences the neck-down profile and a very thin glass fiber is continuously pulled at high fiber drawing speed (Murata, 1996; Paek, 1999). The drawn glass fiber leaves the furnace and enters the subsequent in-line processes that include fiber cooling by helium injection, double layer liquid wet-on-wet resin coatings on fiber surface, and UV curing of coatings.

Fiberoptics industries have been trying to increase both fiber drawing speed and preform size in order to improve the manufacturing productivity of optical fibers. In the early stages of optical fiber mass production in late 1970s and 
early 1980s, fiber drawing speed was around one hundred meters per minute (mpm) and the preform diameter was merely one centimeter or so (Paek and Runk, 1978). At the present time, fiber drawing speed and preform size are now exceeding $2000 \mathrm{mpm}$ and $10 \mathrm{~cm}$, respectively, thanks to technological advances in many aspects of optical fiber manufacturing. The industries can greatly benefit from using preforms of larger diameter, so they could deliver more production of optical fibers per each preform without changing it and restarting manufacturing process so often, and thus significantly reduce the down time of facilities (Boll and Nummela, 2010). However, the increase in preform diameter along with sized up furnace may lead to several problems associated with maintaining radially uniform preform heating and melting of larger preform volume, forming of stable neck-down profiles within acceptable range of draw tension, and keeping the furnace purge gas in stable laminar flow. These problems can cause degradation of optical and mechanical qualities of drawn glass fiber, not to mention the possible breakage of glass fiber during the optical fiber drawing process.

In order to help optimize the draw furnace design and drawing process, there have been various analytical and computational studies on the glass fiber drawing phenomena. Paek and Runk (1978) and Paek (1999) established a one-dimensional momentum balance model for the prediction of preform neck-down profile but the effects of purge gas in draw furnace was treated simply by prescribing convective heat transfer coefficient estimated from the experimental data. Also, there have been multi-dimensional computational investigations on glass fiber drawing by considering the convective transport of purge gas around the preform neck-down shapes which profiles were taken from the measurements (Lee and Jaluria, 1997; Choudhury and Jaluria, 1998; Yan and Pitchumani, 2006).

Later computational research efforts adopted more sophisticated computational schemes for the prediction of neck-down profile formation which is interactively associated with the two-dimensional axisymmetric thermal modeling of radiative preform heating and convective flow of purge gas inside the draw furnace (Cheng and Jaluria, 2005; Chen and Jaluria, 2009; Lee et al., 2006; Mawardi and Pitchumani, 2010; Kim et al,, 2012). The other approach in predicting neck-down profile was to treat the glass preform deformation as free surface flow which profile was determined by kinematic condition (Wei et al, 2004). In addition to developing the efficient numerical methods, some computational investigations focused on several important process characteristics such as effects of draw furnace geometry (Cheng and Jaluria, 2002), process optimization by various design parameters (Cheng and Jaluria, 2005), effects of adding dopant (Yan and Pitchumani, 2006; Chen and Jaluria, 2009), and the role of helium addition in argon-based purge gas (Kim et al,, 2012).

However, the glass fiber drawing conditions in most of previous studies are naturally out of date when viewed from recent industry standards of optical fiber manufacturing by having considered fiber drawing speed and preform diameter well less than $20 \mathrm{~m} / \mathrm{s}$ (or $1200 \mathrm{mpm}$ ) and $10 \mathrm{~cm}$, respectively. Specifically, the preform diameter in the previous theoretical and computational studies of glass fiber drawing has been limited to less than $5 \mathrm{~cm}$ in $1990 \mathrm{~s}$ (Imoto et al., 1989; Lee and Jaluria, 1997; Choudhury and Jaluria, 1998) and less than $10 \mathrm{~cm}$ in 2000s (Cheng and Jaluria, 2005; Wei et al., 2004; Kim et al., 2012). Therefore, it would be necessary to investigate the size effects of enlarged preform on every aspect of drawing process (such as formation of neck-down profile, draw tension, fiber cooling) as well as the validity of modeling approaches, while the fiberoptics industries are constantly demanding stable process techniques with larger sized preform at higher drawing speed.

In this computational study, glass fiber drawing process in an optical fiber draw furnace is numerically modeled and simulated to examine the cases of using a larger sized preform up to $20 \mathrm{~cm}$ diameter at high fiber drawing speed of $30 \mathrm{~m} / \mathrm{s}$ or $1800 \mathrm{mpm}$. The effects of diameter increase in preform as well as of the size-up of draw furnace are investigated on several important aspects in glass fiber drawing such as the formation of preform neck-down profile and fiber draw tension.

\section{Numerical Modeling and Computational Methods 2.1 Simplified model of draw furnace}

Although the actual draw furnace geometry may be quite complex, it has been greatly simplified in present study, as illustrated in Fig. 1. However, this simplified furnace model still possesses all the essential features of draw furnace. It is usual and necessary to place a long thin-walled muffle tube between cylindrical silica preform and graphite resistance heating element of annular cylinder shape. Therefore, preform heating by resistance heater is indirect in order to prevent the undesirable particle contamination coming from possible overheating of heating element and outer 


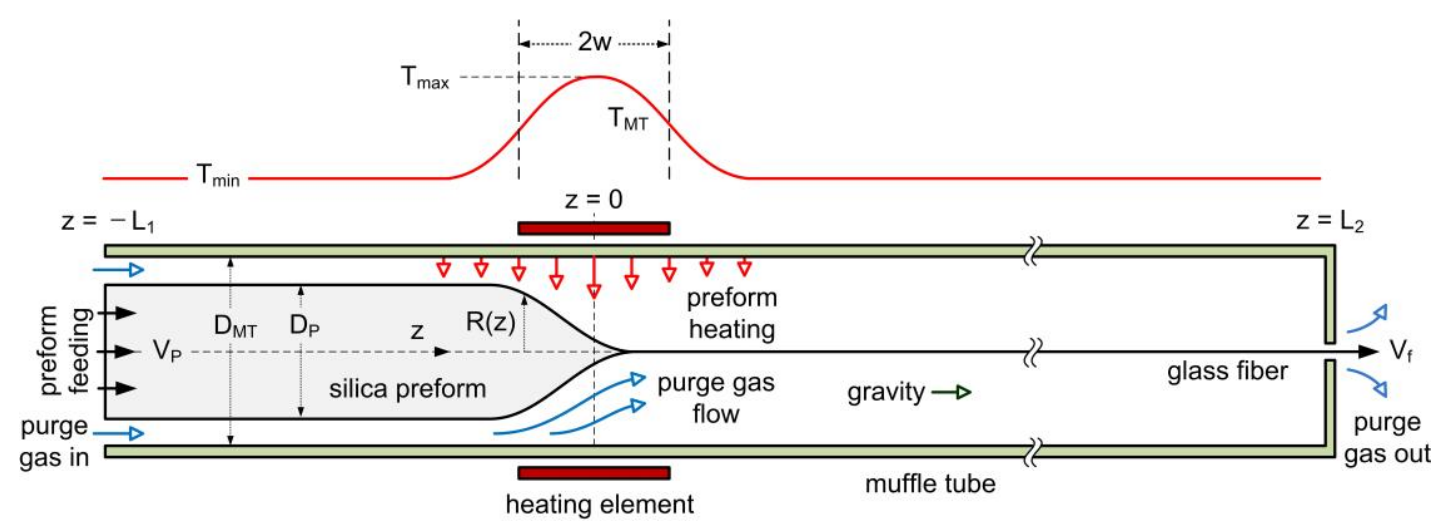

Fig. 1 Schematic of glass fiber drawing from silica preform in draw furnace of optical fiber manufacturing system.

manufacturing environments (Imoto et al, 1989; Kaiser, 1997).

The muffle tube section from tube inlet to the heating zone around resistance heater contains the one or two meters long preform which is fed into the muffle tube slowly. The preform is heated and softened under intense radiative heating in the heating zone and the glass fiber is drawn from the melted tip of neck-down profile by applying adequate amount of fiber draw tension. In lower section of muffle tube, which is sometimes referred as bottom extension tube, a drawn glass fiber is required to be cooled down gradually back to and below glass softening temperature, since unnecessarily high cooling rate is related to increase of Rayleigh scattering and transmission loss in silica based optical fibers (Kim and Tomozawa, 2001; Lancry et al., 2012). Thus, the bottom extension tube is generally long enough to ensure low cooling rate of drawn glass fiber. In this study, total length of muffle tube is set to be 3 meters $\left(L_{1}+L_{2}=1+\right.$ $2 \mathrm{~m})$.

While the target glass fiber diameter at the furnace exit is industry standard $125 \mu \mathrm{m}$, feeding preform diameters ranging from $10 \mathrm{~cm}$ to $20 \mathrm{~cm}$ are tested in this study in order to appreciate the effects of enlarging preform size in glass fiber drawing process. As for the muffle tube, its diameter is also varied to accommodate the increased preform size and the effects of variable gap distance between muffle tube and preform surfaces are also investigated.

The purge gas is supplied into the inner space of draw furnace, as depicted in Fig. 1, and it could play an important role in glass fiber drawing process. It is usually composed of mixture of inert gases such argon or helium and sometimes nitrogen to minimize the oxidation of preform surface and furnace components and clear the contamination particles out of the furnace. Purge gas exits draw furnace with the moving glass fiber through the small iris of $5 \mathrm{~mm}$ diameter at the bottom of muffle tube.

\subsection{Prediction of neck-down profile in silica preform}

The neck-down profile refers to drastic diameter change in softened region of preform which is subjected to local radiative heating and continuous glass fiber drawing. Because the shape of neck-down profile heavily depends on the heating and drawing conditions in draw furnace as well as silica glass properties, the glass fiber drawing is challenging to be numerically simulated due to the complexity of the phenomena. Therefore, present study employs the following simplistic steady-state model of one dimensional (1D) momentum balance in axial direction for the prediction of neck-down profile (Paek, 1999).

$$
\rho \frac{d}{d z}\left(R^{2} v^{2}\right)=\frac{d}{d z}\left(3 R^{2} \mu(T) \frac{d v}{d z}\right)+\rho g R^{2}
$$

Here, $\rho$ and $\mu$ are mass density and viscosity of silica glass, respectively. This equation considers balance between viscous and gravitational forces (on right-hand side) along with the axial momentum advection (on left-hand side) on silica glass flow. Although viscous glass flow in continuous stretching of molten glass could exhibit viscoelastic behavior in general, it is assumed in this study that molten glass flow is Newtonian (Yarin et al., 1999; Uhlmann and Kreidl, 1984; Paek and Runk, 1978). Lu (1999) investigated the effect of viscoelasticity in glass fiber drawing 
simulations. When comparing viscoelastic Maxwell and Jeffrey models with Newtonian model, he found that those models made little difference in computational results, since most of deformation occurs at high temperature where glass viscosity is very low and Newtonian viscous flow dominates. The effect of surface tension on the free surface of preform neck-down profile is expected to be negligible due to high silica viscosity. The reference point of axial coordinate $z$ is set to be the center of heating zone and the computational domain is between both ends of muffle tube $\left(-L_{1}<z<L_{2}\right)$. The gravitational constant $\mathrm{g}$ applies to the direction of glass fiber drawing, as it is consistent with vertical set-up of optical fiber draw tower. In Eq. (1), $R(z)$ and $v(z)$ are preform radius and axial flow velocity in preform, respectively. By the mass conservation, their relation can be expressed as

$$
R(z)=R_{f} \sqrt{V_{f} / v(z)}
$$

with the specified fiber draw speed $V_{f}$ and glass fiber radius $R_{f}$ at the furnace exit. The preform feeding speed $V_{p}$ at the furnace inlet can be easily determined with the given preform feeding radius $R_{p}$. The volumetric feeding rate of preform is defined as

$$
Q=\pi R^{2} v=\pi R_{p}^{2} V_{p}=\pi R_{f}^{2} V_{f}
$$

and, at any axial location, it should be constant under the assumption of steady process for a given glass fiber drawing condition. Therefore, the boundary conditions at both ends of muffle tube for the above governing equation can be given as $v=V_{p}$ at $z=-L_{1}$ and $v=V_{f}$ at $z=L_{2}$.

Although several investigations (Paek, 1999; Lee et al., 2006) which have adopted Eq. (1) included the advection term in the prediction of neck-down profile, this study finds that its effect on the shape of neck-down profile and fiber draw tension is minimal and neglects it for modeling simplicity. This approximation will be verified later by evaluating its effect on draw tension. Thus, by incorporating Eq. (3) into Eq. (1), the governing equation without the advection term becomes

$$
\frac{3}{\rho} \frac{d}{d z}\left(\frac{\mu(T)}{v} \frac{d v}{d z}\right)=-\frac{g}{v}
$$

Once integrating Eq. (4) twice from $-L_{1}$ to $z$ and applying the aforementioned boundary conditions, the axial velocity of silica glass flow in preform can be expressed as

$$
v(z)=V_{p}-\frac{\rho g}{3} \int_{-L_{1}}^{z} \frac{v}{\mu(T)}\left[\int_{-L_{1}}^{z} \frac{d z}{v}\right] d z+\frac{C_{1}}{3} \int_{-L_{1}}^{z} \frac{v}{\mu(T)} d z
$$

where the constant $C_{1}$ is

$$
C_{1}=\frac{V_{f}-V_{p}+\frac{\rho g}{3} \int_{-L_{1}}^{L_{2}} \frac{v}{\mu(T)}\left[\int_{-L_{1}}^{z} \frac{d z}{v}\right] d z}{\frac{1}{3} \int_{-L_{1}}^{L_{2}} \frac{v}{\mu(T)} d z}
$$

Note that this solution is in the implicit form and it requires iteration to determine the neck-down profile along with additional information on axial temperature distribution of preform $T(z)$ for temperature dependent silica glass viscosity. After that, the neck-down profile in preform can be found using Eq. (2). In this approach, one important assumption is the negligible temperature variation of preform in radial direction, and it should be verified later, especially for the simulation of glass fiber drawing from large diameter preform.

\subsection{Temperature dependent viscosity of silica glass}

Viscosity of silica glass varies heavily with temperature and the formation of neck-down profile occurs mainly 


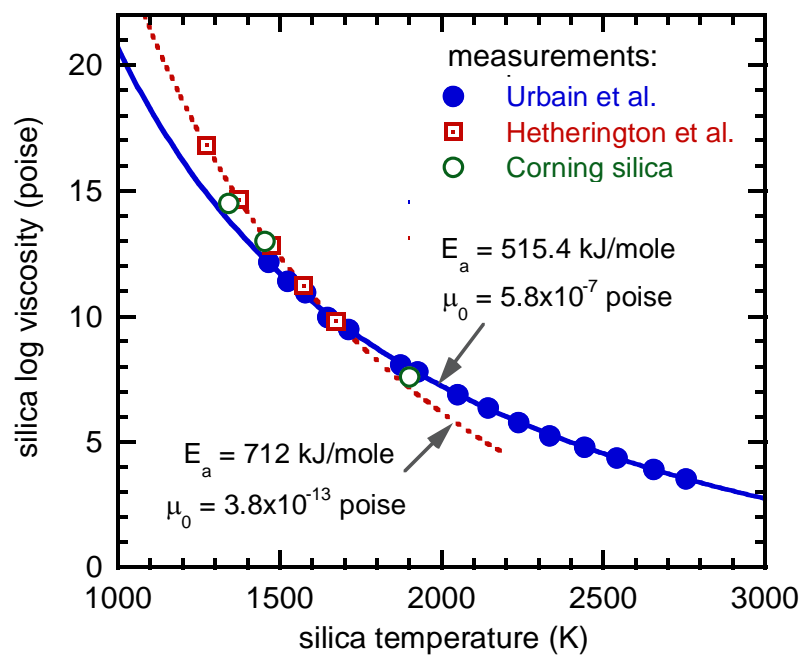

Fig. 2 Temperature dependent silica viscosity: curve fit equations (dotted and solid lines) compared with experimental measurements (circular and square symbols).

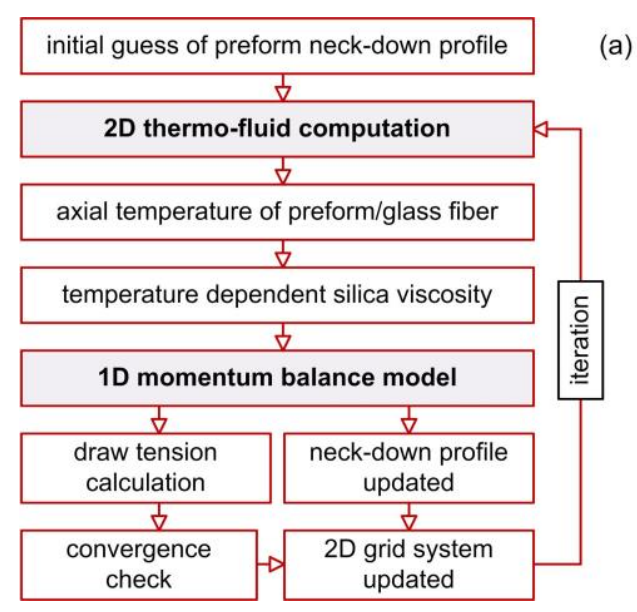

(a)

muffle tube

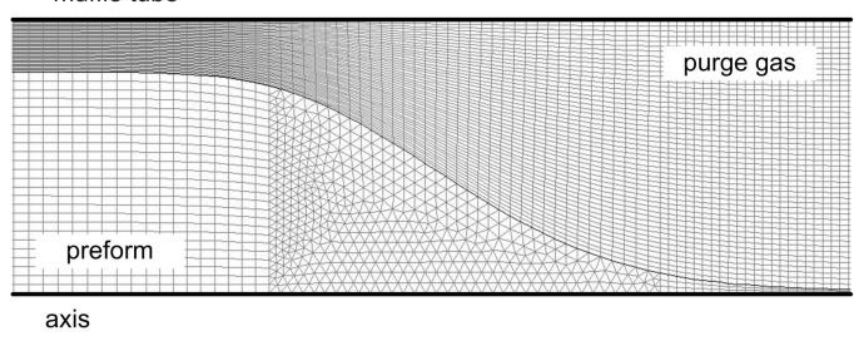

Fig. 3 (a) Iterative computational scheme between 1D and 2D models for the present glass fiber drawing simulations. (b) Computational hybrid grid system for two-dimensional thermo-fluid analysis (case A-1: $D_{p}=10 \mathrm{~cm}, D_{M T}=12.3 \mathrm{~cm}$ ).

because of this drastic change in glass viscosity by the local heating of preform. This study uses the following relationship of Arrhenius type for the silica viscosity (in poise) as a function of temperature ( $T$ in $\mathrm{K}$ ) (Höland and Beall, 2012):

$$
\mu(T)=\mu_{0} \exp \left(\frac{E_{a}}{R T}\right)
$$

where $E_{a}$ an $R$ are the activation energy for viscous flow process and the universal gas constant, respectively.

Doremus (2002) examined many experimental investigations on silica viscosity and concluded that the most reliable data for the temperature range between 1400 and $2500^{\circ} \mathrm{C}$ was from the measurements by Urbain et al. (1982). As shown in Fig. 2, this gives $E_{a}=515.4 \mathrm{~kJ} / \mathrm{mole}$ and $\mu_{0}=5.8 \times 10^{-7}$ poise. The measurements of Hetherington et al. (1964) may be a little more accurate at temperature between 1000 and $1400^{\circ} \mathrm{C}$ and it gives $E_{a}=712 \mathrm{~kJ} / \mathrm{mole}$ and $\mu_{0}=$ $3.8 \times 10^{-13}$ poise. Still, viscosity fit of Eq. (7) with data by Urbain et al. (1982) has been adopted for entire temperature range in present study, since silica viscosity well below softening point is already large enough not to affect the shape of neck-down profile. Fig. 2 also includes the viscosity data for fuzed silica glass of industry grade (Corning Glass 
Code: 7979 and 8655), and they match well with other measurements and fitting equation mentioned above.

\subsection{Iterative computational methods}

In order to determine the neck-down profile with Eq. (5), axial distribution of preform temperature $T(z)$ is required for temperature dependent silica viscosity, and it is obtained from two-dimensional (2D) axisymmetric thermo-fluid computations on heated preform and purge gas flow inside the muffle tube. The numerical scheme may start with 2D computation using the arbitrary initial guess of the preform neck-down profile for the given fiber drawing speed and heating condition. Newly calculated preform and glass fiber temperature along the axis from 2D computational results is then fed into aforementioned 1D model of Eq. (5) to update the neck-down profile of preform. Then, with this updated neck-down profile, the computational domains of purge gas and preform are rearranged and next 2D computation is carried out to determine the preform and glass fiber temperature distribution again. This iterative procedure between 1D and 2D models may proceed until a certain convergence criteria are met, as shown in Fig. 3(a). As the draw tension at the furnace exit is used for checking the convergence here, it is found that approximately ten to fifteen iterations are sufficient and the convergence is quite insensitive to the selection of initially guessed preform neck-down profile.

As illustrated in Fig. 1, the indirect heating of preform by the outer heating element through muffle tube is approximated by prescribing the inner surface temperature of muffle tube with the following form of Gaussian distribution

$$
\frac{T_{M T}(z)-T_{\min }}{T_{\max }-T_{\min }}=\exp \left(-\frac{\left(z-z_{0}\right)^{2}}{2 w^{2}}\right)
$$

where $T_{\max }$ and $T_{\min }$ are maximum and minimum surface temperatures of muffle tube, respectively, given as $T_{\max }=$ $2300 \sim 2900 \mathrm{~K}$ and $T_{\min }=300 \mathrm{~K}$, and $z_{0}$ is the center location of heating zone. The heating zone width is defined as $2 w$, implying one standard deviation width in normal distribution curve. The surface to surface (S2S) thermal radiation is considered between muffle tube and preform/glass fiber surfaces. However, the effect of gas radiation is neglected, since the purge gas consists of monatomic gases such as argon and helium.

For the 2D computation on convective momentum and energy transport of purge gas inside muffle tube, temperature dependent properties of the purge gas such as viscosity and thermal conductivity are utilized, since the temperature variation in purge gas is significant. The silica preform is also treated as the viscous flow but its viscosity is deliberately given a very large value for the computational convenience, not using Eq. (7), because the viscosity of silica even at high temperature is expected to be far larger than the one of purge gas. As the flow boundary condition at the surface of preform and glass fiber domain, the interface velocity for each side of preform and purge gas is given from the solution obtained in 1D formulation for each iteration between 1D and 2D models. At this interface, thermal boundary condition is the continuity in temperature and convective heat flux. Both of purge gas and silica flows are assumed to be steady and laminar.

The transmission and absorption of radiative energy in optically transparent silica preform is estimated by adopting apparent thermal conductivity with Rosseland diffusion approximation and its effect is added into effective thermal conductivity of silica glass (Paek, 1999; Choudhary et al., 2010). Molecular thermal conductivity of silica glass is merely $2.68 \mathrm{~W} / \mathrm{m} \cdot \mathrm{K}$, but this radiative transmission and absorption into preform enables radially uniform preform heating much more effectively. The surface emissivity of the preform and glass fiber is set to be constant at 0.885 (Yan and Pitchumani, 2006). Aforementioned 2D thermo-fluid analysis is carried out for two separate domains of the purge gas and the axially moving preform and glass fiber using the general purpose CFD software, ANSYS FLUENT.

\subsection{Calculation of fiber draw tension}

It is important to maintain a proper amount of draw tension on glass fiber. Too high draw tension may cause the fiber breakage, or, if it is too low, it could be the source of undesirable fiber vibration and process instability in the continuous drawing process. The strength of draw tension recommended in fiberoptics industries is known to be on the order of $100 \mathrm{~g}$ or $1 \mathrm{~N}$. By considering the force balance between draw tension and viscous and gravitational forces, 
draw tension acting on the drawn glass fiber or preform at a given arbitrary z location can be evaluated as (Choudhury and Jaluria, 1998)

$$
F_{T}(z)=3 \pi \mu R^{2} \frac{d v}{d z}-\pi \rho g \int_{z}^{L_{2}} R^{2} d z
$$

Providing Eq. (3) and the first integral form of Eq. (4) into Eq. (9) gives the following form of more convenient expression for estimating fiber draw tension using pre-calculated constant $C_{1}$ in Eq. (6) and axial velocity distribution in glass fiber and preform.

$$
F_{T}=C_{1} Q-\rho g Q \int_{-L_{1}}^{L_{2}} \frac{d z}{v}
$$

\section{Computational Results and Discussion 3.1 Model description and verification}

In this computational study, the following optical fiber drawing conditions are given based on the actual optical fiber manufacturing process in a typical optical fiber draw furnace at the present time. First, the fiber drawing speed is given and fixed at $30 \mathrm{~m} / \mathrm{s}$ or $1800 \mathrm{mpm}$ at draw furnace exit. The purge gas is the mixture of helium and argon gase $\mathrm{s}$ with the equal volume ratio and its supply rate is 10 liters per minute with the inlet gas temperature of $300 \mathrm{~K}$ at top end of muffle tube. The thermophysical properties of this binary gas mixture required for $2 \mathrm{D}$ convective transport computations are evaluated using the semi-empirical formula of Wilke (Bird et al., 2001). The pressure outlet condition is given at small iris exit of muffle tube bottom.

Table 1 lists total five geometrical draw furnace cases of different muffle tube and silica preform sizes which are numerically simulated here in this investigation. For three feeding preform diameters of 10, 15, and $20 \mathrm{~cm}$, muffle tube diameter is either chosen with the fixed gap between preform and muffle tube $\left(D_{M T}=12.3,17.3,22.3 \mathrm{~cm}\right.$ for $D_{p}=10$, $15,20 \mathrm{~cm}$, respectively, as in cases A-1, B-1, and C) or with the fixed size $\left(D_{M T}=22.3 \mathrm{~cm}\right.$ for all preform diameters as in cases A-2, B-2, and C). Note that cases A-2 and B-2 are designated for possibly necessary occasion that smaller sized preforms are tried in large sized muffle tube, because exchange of muffle tube is difficult once installed in optical fiber draw tower. As in actual manufacturing system, muffle tube of $3 \mathrm{~m}$ length is relatively long compared to its diameter with cylindrical aspect ratio approximately from 13.5 to 24.4 .

Fig. 3(b) shows the example of hybrid grid system used in 2D axisymmetric FLUENT computations in the vicinity of heating zone and preform neck-down profile for case A-1 of $10 \mathrm{~cm}$ diameter preform and $12.3 \mathrm{~cm}$ diameter muffle tube. This grid system employs the structured quadrilateral meshes for gas domain and preform and glass fiber domain, but the neck-down region of preform uses the unstructured triangular meshes. The total number of computational meshes in 2D thermo-fluid analysis varies depending on the preform size and it is 52,400, 78,800, and 104,800 for the cases of $D_{p}=10,15,20 \mathrm{~cm}$, respectively. Preform temperature at the inlet is $300 \mathrm{~K}$, which seems to be somewhat arbitrary, but it should not affect the simulation accuracy, since the preform length before the heating zone is long

Table 1. Geometric dimensions of draw furnace model and calculated draw tension from glass fiber drawing process simulations.

\begin{tabular}{|c|c|c|c|c|c|c|}
\hline \multirow{2}{*}{ case } & \multirow{2}{*}{$\begin{array}{c}\text { preform } \\
\text { diameter } \\
D_{p}(\mathrm{~cm})\end{array}$} & $\begin{array}{c}\text { muffle tube } \\
\text { diameter } \\
D_{M T}(\mathrm{~cm})\end{array}$ & $\begin{array}{c}T_{\max }=2500 \mathrm{~K} \\
\text { (present) } \\
\text { w/o advection } \\
\text { w/ gravity }\end{array}$ & $\begin{array}{c}\text { fiber draw tension } F_{T}(\mathrm{~g}) \\
\text { w/o advection } \\
\text { w/ gravity }\end{array}$ & $\begin{array}{c}\text { (prest) } \\
\text { w/ advection } \\
\text { w/ gravity }\end{array}$ & $\begin{array}{c}T_{\max }=2700 \mathrm{~K} \\
\text { w/o advection } \\
\text { w/o gravity }\end{array}$ \\
\hline A-1 & 10 & 12.3 & $\mathbf{2 3 3 . 5}$ & $\mathbf{2 0 . 2}$ & 19.9 & 25.2 \\
\hline B-1 & 15 & 17.3 & $\mathbf{7 7 9 . 9}$ & $\mathbf{5 1 . 1}$ & 50.9 & 62.9 \\
\hline C & 20 & 22.3 & $\mathbf{2 5 9 3 . 1}$ & $\mathbf{1 3 9 . 4}$ & 139.1 & 165.9 \\
\hline B-2 & 15 & 22.3 & $\mathbf{1 3 5 7 . 8}$ & $\mathbf{9 5 . 0}$ & 94.7 & 112.2 \\
\hline A-2 & 10 & 22.3 & $\mathbf{7 6 3 . 5}$ & $\mathbf{6 9 . 1}$ & 68.9 & 79.7 \\
\hline
\end{tabular}


enough. Note that the other geometric figures and prescribed distribution of muffle tube surface temperature have been already stated in previous section of describing simplified model of draw furnace.

For the same case as in Fig. 3(b) (case A-1), converged numerical results from iterative computations between 1D and 2D models are presented in Fig. 4. This case serves for verification purpose, being compared to optical fiber draw furnace tests provided by Samsung Electronics Fiberoptics Division. Reflecting heating element temperature and size in actual tests with $10 \mathrm{~cm}$ diameter preform, heating condition is given that maximum temperature of muffle tube surface is $T_{\max }=2500 \mathrm{~K}$ and heating zone width $2 w$ is $30 \mathrm{~cm}$. Since the muffle tube is being cooled by circulation of cooling water except the heating zone, minimum temperature of muffle tube surface is set to be $T_{\min }=300 \mathrm{~K}$.

Firstly, Fig. 4(a) shows axial temperature distribution along the centerline of preform and drawn glass fiber from muffle tube inlet to exit. Preform temperature increases rapidly around heating zone, where neck-down profile occurs, with its maximum of approximately $2400 \mathrm{~K}$ in the middle of neck-down profile, and glass fiber temperature decreases rather slowly in bottom portion of muffle tube. Secondly, Fig. 4(b) shows a smooth shape of preform neck-down profile, as its length is roughly $15 \mathrm{~cm}$, approximately a half of heating zone width. In this figure, measured shape of neck-down profile is also compared with predicted results, and the agreement is quite favorable. Note that some degree of discrepancy near the end of neck-down profile is due to the fact that measurement was taken from remaining portion of preform after production run and the end shape became disfigured by re-solidification from melted state.

Also, the location of softening temperature for silica glass, which is approximately $1950 \mathrm{~K}$, is estimated to be $z=$ $-9 \mathrm{~cm}$ in Fig. 4(a) and it coincides with the starting location of preform neck-down profile in Fig. 4(b). On the other hand, fiber draw tension is calculated to be $234 \mathrm{~g}$, which is the value on the order of $100 \mathrm{~g}$ found in actual process. Grid sensitivity tests have been also conducted for this case with finer grid systems by doubling mesh numbers either in radial or axial directions. In Figs. 4(a) and (b), there is no discernible difference observed among the numerical results from those three grid systems and it demonstrates that grid independency is ensured in 2D computations. Note that grid sensitivity was also tested and validated in the same way for the case with larger preform diameter of $20 \mathrm{~cm}$, though not shown here.

\subsection{Effects of varying preform and muffle tube diameters}

In Fig. 5, two-dimensional computational results of temperature and axial velocity contours as well as streamline patterns of purge gas flow can be seen for all five cases listed in Table 1 under the identical heating condition of $T_{\max }=$ $2700 \mathrm{~K}$. In all cases, predicted neck-down shapes of heated preform exhibit a typical profile as observed in actual optical fiber drawing process. The streamlines of slowly flowing purge gas inside muffle tube indicate quite a simple laminar flow pattern around the neck-down profile with axial velocity magnitude of well less than $0.5 \mathrm{~m} / \mathrm{s}$. There is not
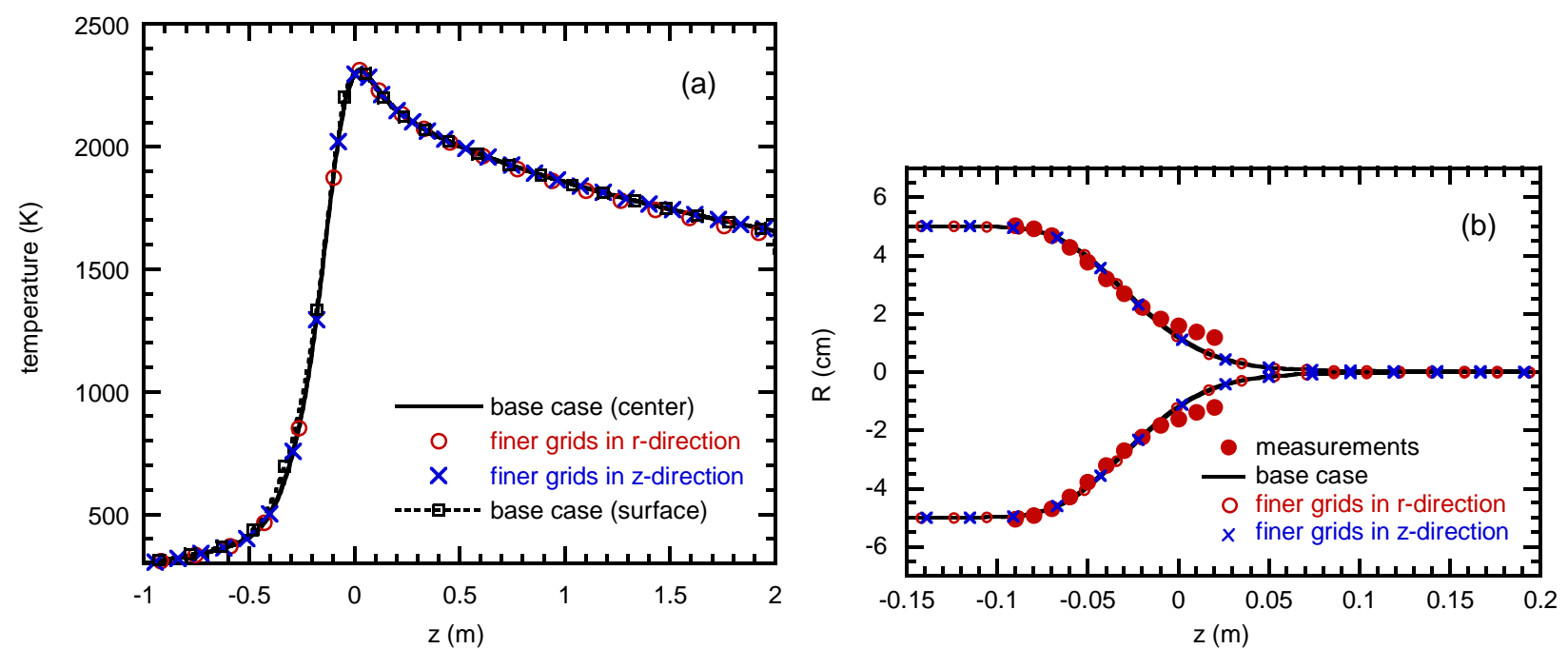

Fig. 4 Validation and grid sensitivity tests on (a) axial temperature distribution in preform and glass fiber and (b) preform neck-down profile for the drawing condition of $V_{f}=30 \mathrm{~m} / \mathrm{s}$ and $T_{\max }=2500 \mathrm{~K}$ (case A-1: $D_{p}=10 \mathrm{~cm}, D_{M T}=12.3 \mathrm{~cm}$ ). Solid symbols represent measured neck-down profile for same condition. 

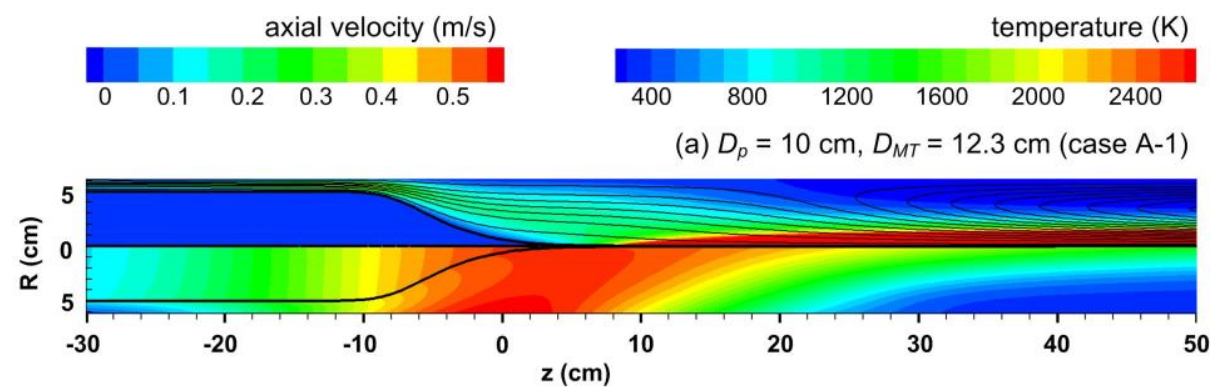

(b) $D_{p}=15 \mathrm{~cm}, D_{M T}=17.3 \mathrm{~cm}$ (case B-1)

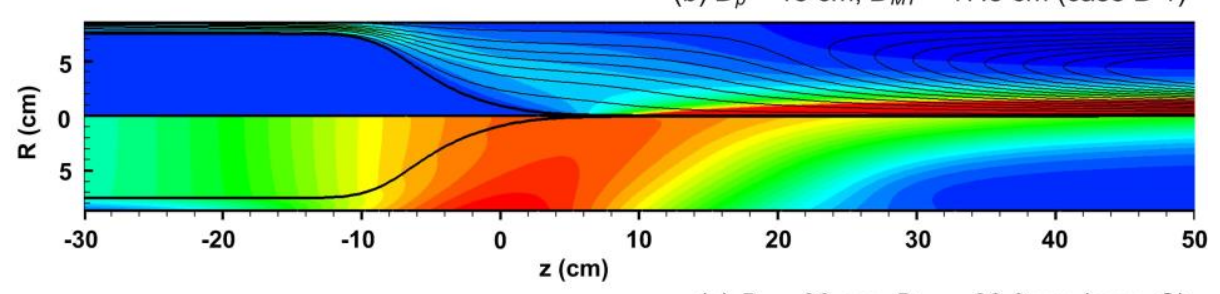

(c) $D_{p}=20 \mathrm{~cm}, D_{M T}=22.3 \mathrm{~cm}$ (case C)

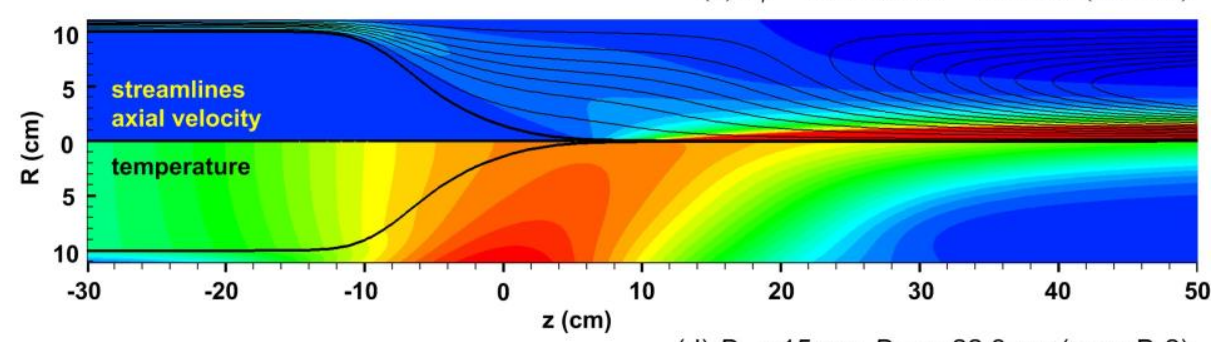

(d) $D_{p}=15 \mathrm{~cm}, D_{M T}=22.3 \mathrm{~cm}($ case B-2)

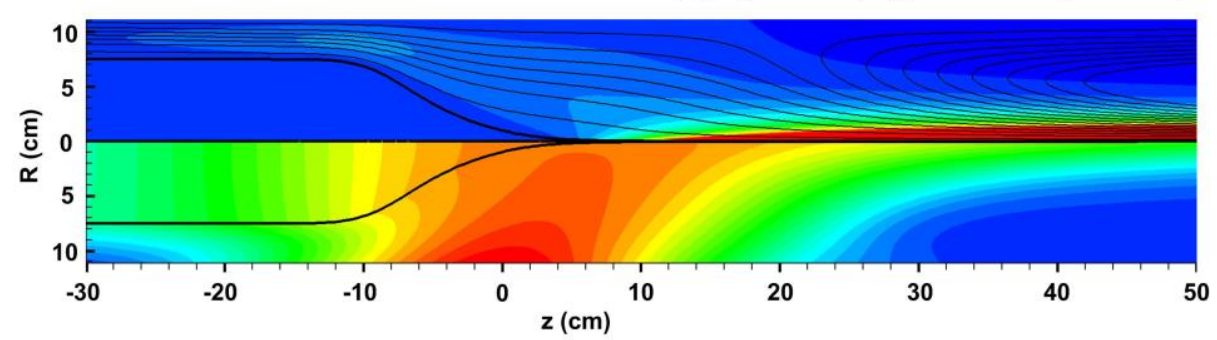

(e) $D_{p}=10 \mathrm{~cm}, D_{M T}=22.3 \mathrm{~cm}($ case A-2)

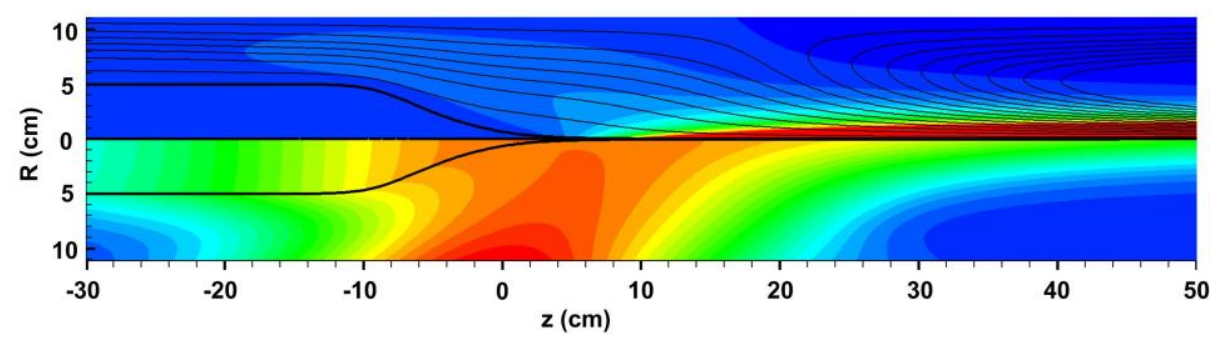

Fig. 5 Streamline patterns with axial velocity contours (upper) and temperature contours (lower) in heated preform and purge gas flow $\left(V_{f}=30 \mathrm{~m} / \mathrm{s}, T_{\max }=2700 \mathrm{~K}\right)$.

any sign of flow separation when purge gas passes down the neck-down shape even for the case of large diameter preform. In fact, this type of simple and smooth flow pattern is favored in draw furnace for maintaining process stability in high precision optical fiber manufacturing. Starting around $z=10 \mathrm{~cm}$, shear flow layer appears over the drawn glass fiber and convective cooling of glass fiber becomes significant, as glass fiber is drawn from the tip of neck-down shape and its speed rapidly approaches given fiber drawing speed of $30 \mathrm{~m} / \mathrm{s}$. At the same time, the flow recirculation starting from $z=25 \mathrm{~cm}$ occurs because of closed end of muffle tube with the small iris opening where the glass fiber and purge gas exit the furnace. This recirculation of purge gas could be the cause for undesirable accumulation of contamination particles within draw furnace. 

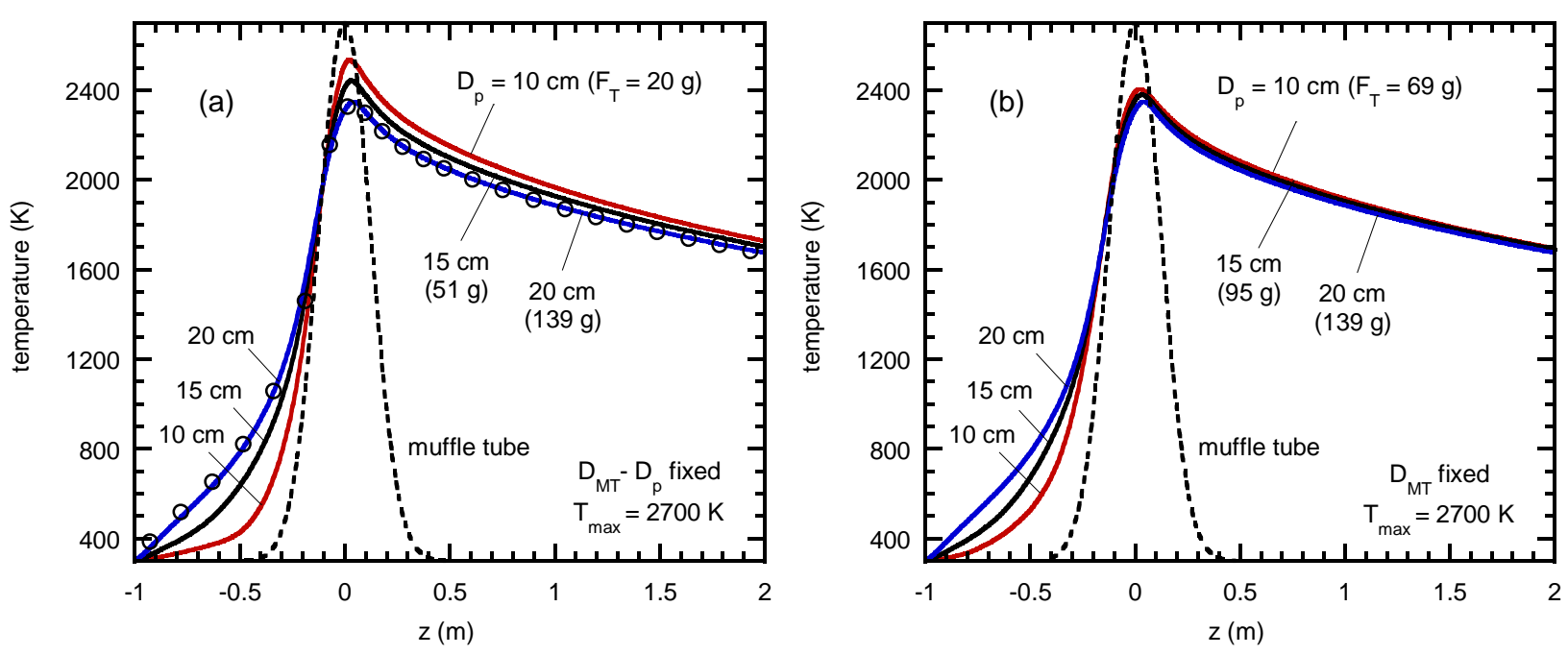

Fig. 6 Effects of silica preform size on axial temperature distribution in preform and glass fiber $\left(V_{f}=30 \mathrm{~m} / \mathrm{s}, T_{\max }=2700\right.$ $\mathrm{K})$ : (a) cases with fixed gap distance between preform and muffle tube; (b) cases with fixed muffle tube diameter. Symbols represent surface temperature of preform and glass fiber for case of $D_{p}=20 \mathrm{~cm}$.
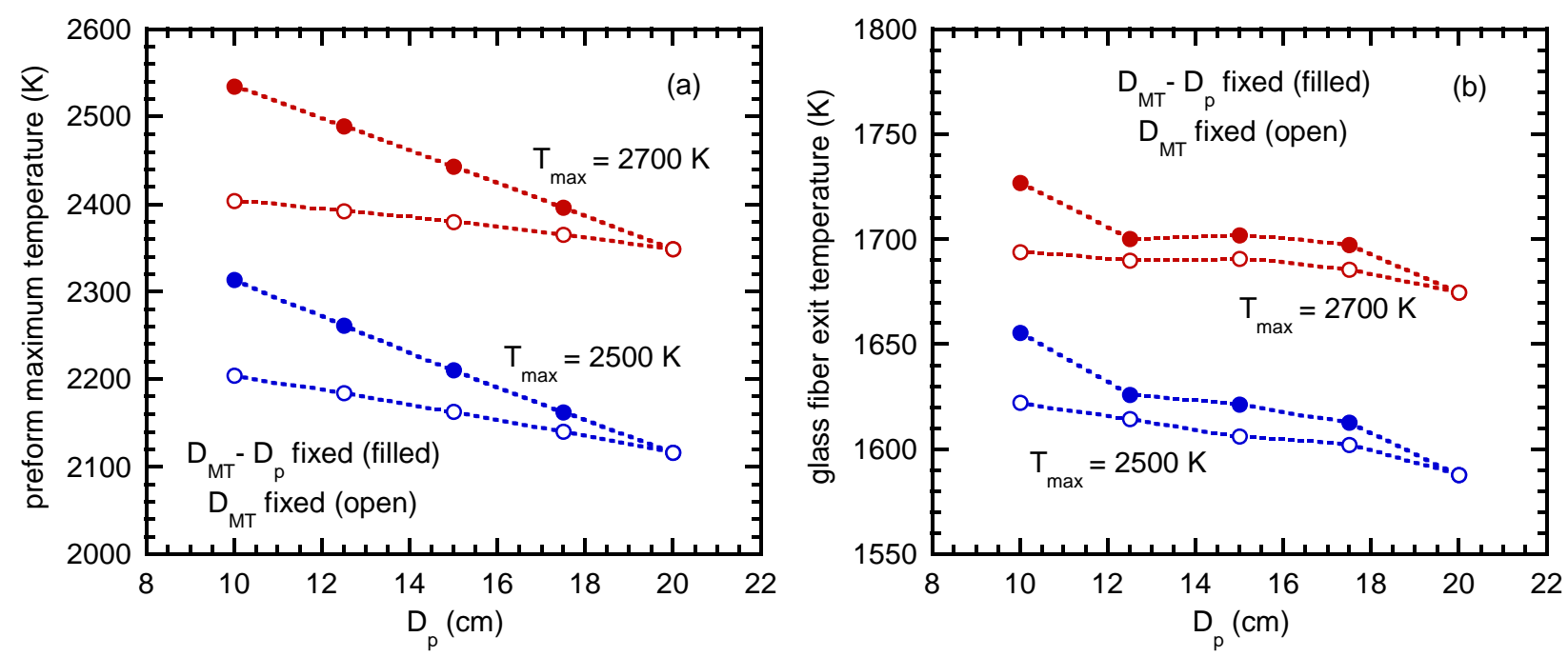

Fig. 7 Effects of silica preform size on preform maximum temperature and glass fiber exit temperature for two heating conditions of $T_{\max }=2500$ and $2700 \mathrm{~K}\left(V_{f}=30 \mathrm{~m} / \mathrm{s}\right)$.

The effects of varying preform and muffle tube diameters are shown in Fig. 6 on axial temperature distribution along the centerline of preform and glass fiber for heating condition of $T_{\max }=2700 \mathrm{~K}$, also shown is the prescribed surface temperature of muffle tube. Fig. 6(a) shows the cases same as in Figs. 5(a), (b), and (c), while Fig. 6(b) includes the cases shown in Figs. 5(c), (d), and (e). As all cases exhibit a similar trend of temperature distribution, maximum temperature that occurs in heated portion of preform is important, since it is directly related to draw tension, and it is summarized in Fig. 7(a) for two heating conditions of $T_{\max }=2500$ and $2700 \mathrm{~K}$.

Maximum preform temperature decreases with larger preform diameter, but it is so less considerably when muffle tube diameter is fixed. Fig. 7(b) gives glass fiber temperature at furnace exit, and it is found to be well below silica softening temperature of $1950 \mathrm{~K}$ for all cases. Although the cases with fixed muffle tube diameter show marginally lower exit glass fiber temperature, size effects are found to be relatively insignificant. This shows the necessity of long extension in muffle tube after heating zone. After draw furnace, there usually exists a dedicated glass fiber cooling unit with helium injection where main cooling of fast moving glass fiber close to room temperature, just before sufficiently cooled glass fiber enters surface coating process (Vaskopulos et al., 1995; Yang et al, 2008; Kim et al., 2011). Still, it is important to cool down glass fiber below softening point at furnace exit, since uncoated glass fiber above softening point is expected to be more prone to particle contamination and damage on its surface in outer environments. 


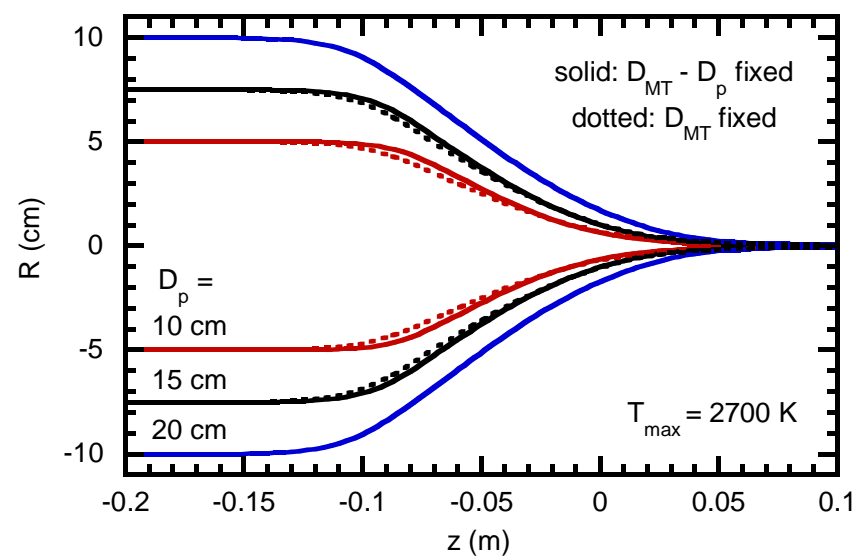

Fig. 8 Effects of silica preform size on preform neck-down profile $\left(V_{f}=30 \mathrm{~m} / \mathrm{s}, T_{\max }=2700 \mathrm{~K}\right)$. Solid lines are for cases with fixed gap distance between preform and muffle tube, while dotted lines are for cases with fixed muffle tube diameter.

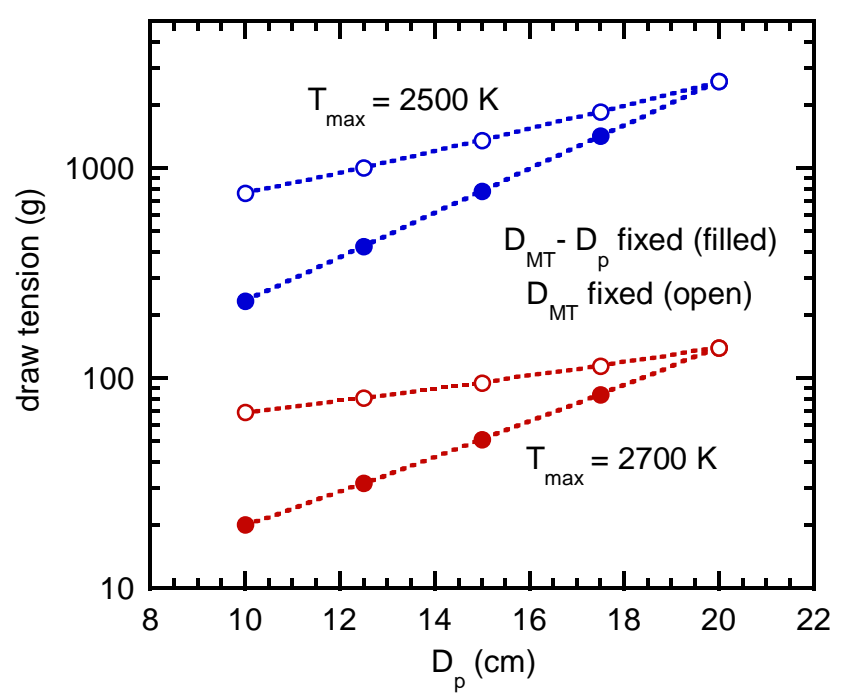

Fig. 9 Effects of silica preform size on glass fiber draw tension for two heating conditions of $T_{\max }=2500$ and $2700 \mathrm{~K}\left(V_{f}=\right.$ $30 \mathrm{~m} / \mathrm{s})$.

Fig. 6(a) additionally contains preform surface temperature for the case of $D_{p}=20 \mathrm{~cm}$ (expressed as circular symbols in the figure) and it is found that difference between centerline and surface temperature in preform is effectively negligible, especially compared to axial temperature gradient. This verifies the assumption of radially uniform temperature in neck-down region, as mentioned previously in discussing 1D prediction model for neck-down profile. Therefore, 1D approximation of 2D preform temperature to axial direction is very valid even for large sized preform. It can be observed and checked with preform temperature contours of Fig. 5 for all cases, or again with surface and inner temperature comparison in Fig. 4(a) for the case of $D_{p}=10 \mathrm{~cm}$. The preform neck-down shapes among those five cases of glass fiber drawing shown in Figs. 5 and 6 are compared in Fig. 8. The cases of fixed muffle tube diameter show slightly longer neck-down profile, due to more distributed radiative heating by enlarged gap distance between preform and muffle tube surfaces.

\subsection{Analysis of glass fiber draw tension}

The effects of preform and muffle tube sizes on draw tension acting on glass fiber at furnace exit are summarized in Table 1 and also graphically shown in Fig. 9 for two heating conditions of $T_{\max }=2500$ and $2700 \mathrm{~K}$. As mentioned 

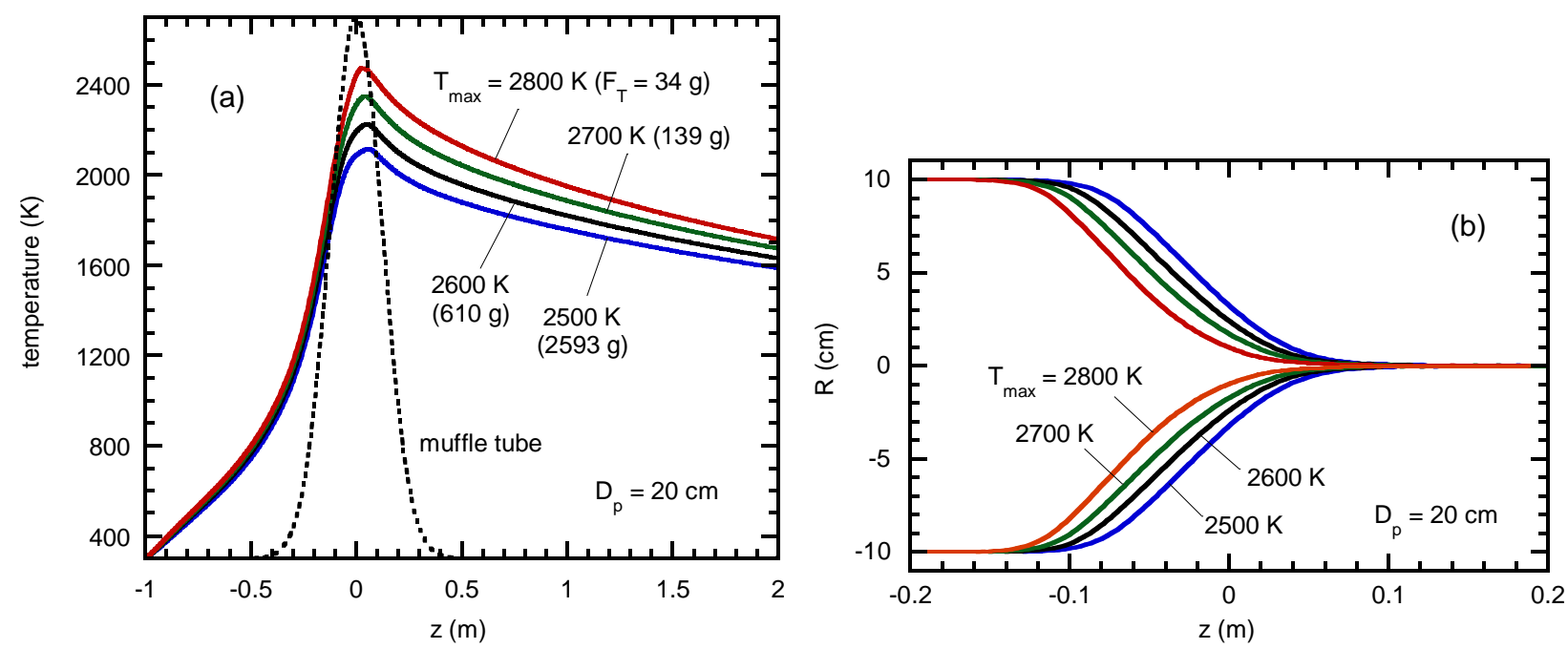

Fig. 10 Effects of heating temperature on (a) axial temperature distribution in preform and glass fiber and (b) preform neck-down profile for the drawing condition of $D_{p}=20 \mathrm{~cm}$ and $V_{f}=30 \mathrm{~m} / \mathrm{s}$.

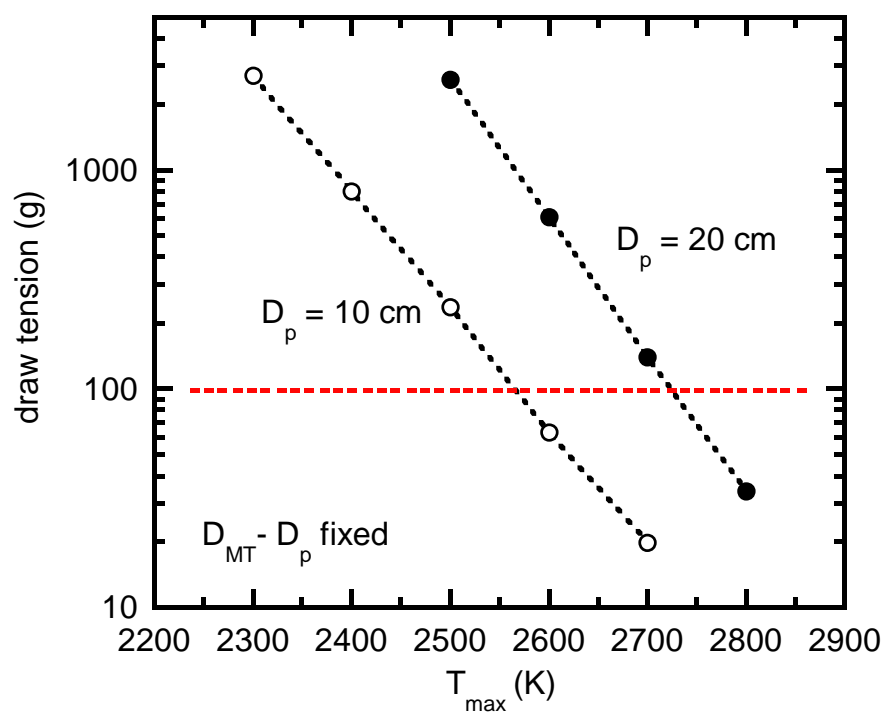

Fig. 11 Effects of heating temperature on glass fiber draw tension for two preform diameters of 10 and $20 \mathrm{~cm}\left(V_{f}=30 \mathrm{~m} / \mathrm{s}\right)$.

previously, 1D prediction model of preform neck-down shape in this investigation considers only viscous and gravitational forces, excluding advection effects of axially moving preform and glass fiber. Table 1 also lists the effects of this assumption on draw tension for $T_{\max }=2700 \mathrm{~K}$ by comparing the present results of draw tension with two different evaluation approaches: (1) calculation that includes all viscous and gravitational forces and also advection term; (2) calculation only with viscous force, excluding both advection effect and gravitational force. It is found that the inclusion of advection term makes very small difference of only $0.2 \sim 0.3 \mathrm{~g}$ force in evaluating draw tension for all cases of computation, and it shows the validity of neglecting advection term in the present model. On the other hand, the effect of gravitational force is relatively significant on draw tension by making difference of 15 to 25 percent. This comparison confirms that viscous force depending heavily with temperature is dominant phenomenon in determining preform neck-down shape and draw tension. Though less accurate, rough estimation of neck-down shape and draw tension is still possible by considering only viscous force, which will further simplify 1D neck-down prediction model.

As shown in Fig. 9, increase in preform diameter as well as larger gap distance between preform and muffle tube leads to higher draw tension, as expected. However, heating temperature change affects draw tension more profoundly, as draw tension decreases more than one-tenth with $200 \mathrm{~K}$ increase of $T_{\max }$. In fact, Fig. 9 of draw tension inversely follows Fig. 7(a) of maximum preform temperature, although draw tension is plotted in logarithmic scale, due to the 
fact that silica viscosity drops exponentially with temperature as in Eq. (7). Thus, draw tension is largely decided by resulting maximum preform temperature around heating zone under given heating condition in geometric setup of preform and muffle tube.

For a large sized preform of $D_{p}=20 \mathrm{~cm}$ (case C), Fig. 10(a) shows elevation of axial temperature of preform and glass fiber by increased heating temperature $\left(T_{\max }=2500 \sim 2800 \mathrm{~K}\right)$, especially in the region of neck-down shape and glass fiber, while the neck-down profile moves upward with increasing heating temperature in Fig. 10(b). Fig. 11 summarizes the effects of heating condition on draw tension for two preform diameters of $D_{p}=10$ and $20 \mathrm{~cm}$ (cases A-1 and C, respectively). It shows significant and sensitive change of draw tension with temperature adjustment of heating element in draw furnace, even if temperature change is small. Since recommended draw tension is around 100 $\mathrm{g}$, proper heating temperature $T_{\max }$ is found to be approximately $2550 \mathrm{~K}$ for preform diameter of $10 \mathrm{~cm}$. When preform diameter is as large as $20 \mathrm{~cm}$, draw tension would be more than $2500 \mathrm{~g}$, which is unacceptably high enough to cause fiber breakage, if heating condition remains unchanged, and heating temperature needs to increase to $2720 \mathrm{~K}$. Due to drastic change of silica viscosity with temperature, these computational results demonstrate that draw furnace requires fine and careful control of heating element in order to maintain proper draw tension in glass fiber drawing process.

\section{Conclusions}

In this computational study, high speed glass fiber drawing from heated and softened silica preform is numerically modeled and investigated by employing the iterative scheme between 1D prediction model for preform neck-down profile and 2D thermo-fluid computations of radiative and convective preform heating in simplified geometry of draw furnace. The major findings from computational results of glass fiber drawing simulations at drawing speed of 1800 mpm are as follows. Applying the actual draw furnace conditions such as muffle tube size, heating element temperature, and supply rate of helium/argon purge gas, present numerical model is verified for glass fiber drawing from $10 \mathrm{~cm}$ diameter preform by showing that predicted neck-down shape and draw tension agree well with corresponding measurements.

Many cases of preform size up to $20 \mathrm{~cm}$ diameter in sized up muffle tube are numerically tested and analyzed to appreciate the effects of preform and muffle tube diameters on axial temperature distribution and neck-down shape. Computational results found that assumption of radially uniform temperature is still valid for large diameter preform heating and, long muffle tube is necessary to cool down drawn glass fiber at furnace exit well below glass softening point. Also, purge gas flow around neck-down shape exhibits stable and smooth patterns without any flow separation, as favored in fiberoptics industries. Fiber draw tension on glass fiber is an important operation parameter in optical fiber manufacturing, and viscous and gravitational forces are found to be dominant components in determining draw tension, while advection effect is negligibly small. Draw tension changes drastically with increased heating temperature and fine control of heating element temperature is necessary. Large sized preform requires higher heating temperature in order to maintain proper amount of draw tension around $100 \mathrm{~g}$.

\section{Acknowledgment}

This research was supported by Basic Science Research Program through the National Research Foundation of Korea (NRF) funded by the Ministry of Education (NRF-2016R1D1A1B03931662).

\section{References}

Bird, R. B., Stewart, W. E. and Lightfoot, E. N., Transport Phenomena, 2nd ed. (2001), Wiley, New York.

Boll, K. and Nummela, J., Large optical fiber drawing furnace developments, Proceedings of 59th International Wire and Cable Symposium, Providence, RI, USA (2010).

Chen, C. and Jaluria, Y., Effects of doping on the optical fiber drawing process, International Journal of Heat and Mass Transfer, Vol.52, No.21-22 (2009), pp.4812-4822.

Cheng, X. and Jaluria, Y., Effect of draw furnace geometry on high-speed optical fiber manufacturing, Numerical Heat Transfer, Part A, Vol.41, No.8 (2002), pp.757-781.

Cheng, X. and Jaluria, Y., Optimization of a thermal manufacturing process: drawing of optical fibers, International 
Journal of Heat and Mass Transfer, Vol.48, No.17 (2005), pp.3560-3573.

Choudhary, M. K., Venuturumilli, R. and Hyre, M.R., Mathematical modeling of flow and heat transfer phenomena in glass melting, delivery, and forming process, International Journal of Applied Glass Science, Vol.1, No.2 (2010), pp.188-214.

Choudhury, S. R. and Jaluria, Y., Thermal transport due to material and gas flow in a furnace for drawing an optical fiber, Journal of Materials Research, Vol.13, No.2 (1998), pp.494-503.

Doremus, R. H., Viscosity of Silica, Journal of Applied Physics, Vol.92, No.12 (2002), pp.7619-7629.

Elliott, B. and Gilmore, M., Fiber Optic Cabling, 2nd ed. (2001), Butterworth-Heinemann, Oxford.

Hetherington, G., Jack, K. H. and Kennedy, J. C., The viscosity of vitreous silica, Physics and Chemistry of Glasses, Vol.5 (1964), pp.130-136.

Höland, W. and Beall, G. H., Glass-Ceramic Technology, 2nd ed. (2012), Wiley, Hoboken.

Imoto, K., Sumi, M., Toda, G. and Suganuma, T., Optical fiber drawing method with gas flow controlling system, Journal of Lightwave Technology, Vol.7, No.1 (1989), pp.115-121.

Kaiser, P., Contamination of furnace-drawn silica fibers, Applied Optics, Vol.16, No.12 (1997), pp.701-704.

Kim, D.-L. and Tomozawa, M., Fictive temperature of silica glass optical fibers - re-examination, Journal of Non-Crystalline Solids, Vol.286, No.1-2 (2001), pp.132-138.

Kim, K., Kwak, H. S. and Kim, D., The role of helium/argon gas flow in a glass fiber drawing furnace, Computational Thermal Sciences, Vol.4, No.3 (2012), pp.263-270.

Kim, K., Kwak, H. S., Park, S. H. and Lee, Y. S., Theoretical prediction on double-layer coating in wet-on-wet optical fiber coating process, Journal of Coatings Technology and Research, Vol.8, No.1 (2011), pp.35-44.

Lancry, M., Régnier, E. and Poumellec, B., Fictive temperature in silica-based glasses and its application to optical fiber manufacturing, Progress in Materials Science, Vol.57, No.1 (2012), pp.63-94.

Lee, K.-M., Wei, Z., Zhou, Z. and Hong, S.-P., Computational thermal models for design of a modern fiber draw process, IEEE Transactions on Automation Science and Engineering, Vol.3, No.1 (2006), pp.108-118.

Lee, S. H. K. and Jaluria, Y., Simulation of the transport processes in the neck-down region of a furnace drawn optical fiber, International Journal of Heat and Mass Transfer, Vol.40, No.4 (1997), pp.843-856.

Lu, X., Experimental and analytical investigation of nonisothermal viscoelastic glass fiber drawing, Ph.D. Dissertation (1999), The University of Michigan.

Mawardi, A. and Pitchumani, R., Optical fiber drawing process model using an analytical neck-down profile, IEEE Photonics Journal, Vol.2, No.4 (2010), pp.620-629.

Murata, H., Handbook of Optical Fibers and Cables, 2nd ed. (1996), Taylor \& Francis, Inc., New York.

Paek, U. C., Free drawing and polymer coating of silica glass optical fibers, Journal of Heat Transfer, Vol.121, No.4 (1999), pp.1-15.

Paek, U. C. and Runk, R. B., Physical behavior of the neck - down region during furnace drawing of silica fibers, Journal of Applied Physics, Vol.49 (1978), pp.4417-4422.

Richardson, K., Krol, D. and Hirao, K., Glasses for photonics applications, International Journal of Applied Glass Science, Vol.1, No.1 (2010), pp. 74-86.

Uhlmann, D. R. and Kreidl, N. J., Glass Science and Technology (1984), Academic Press, New York.

Urbain, G., Bottinga, Y. and Richet, P., Viscosity of liquid silica, silicates and alumino-silicates, Geochimica et Cosmochimica Acta, Vol.46, No.6 (1982), pp.1061-1072.

Vaskopulos, T., Polymeropoulos, C. and Zebib, A., Cooling of optical fiber in aiding and opposing forced gas flow, International Journal of Heat and Mass Transfer, Vol.38, No.11 (1995), pp.1933-1944.

Wei, Z., Lee, K.-M., Tchikanda, S.W., Zhou, Z. and Hong, S.-P., Free surface flow in high speed fiber drawing with large-diameter glass preforms, Journal of Heat Transfer, Vol.126, No.5 (2004), pp.713-722.

Yan, Y. and Pitchumani, R., Numerical study on the dopant concentration and refractive index profile evolution in an optical fiber manufacturing process, International Journal of Heat and Mass Transfer, Vol.49, No.13-14 (2006), pp.2097-2122.

Yang, A., Tao, X. M. and Cheng, X. Y., Prediction of fiber coating thickness via liquid-phase process, Journal of Materials Processing Technology, Vol.202, No.1-3 (2008), pp.365-373.

Yarin, A. L., Gospodinov, P., Gottlieb, O. and Graham, M. D., Newtonian glass fiber drawing: Chaotic variation of the cross-sectional radius, Physics of Fluids, Vol.11, No.11 (1999), pp. 3201-3208. 